\title{
LA POÉSIE DE VERLAINE ET LA POÉSIE CHINOISE DANS LA PERSPECTIVE DE LA RÉCEPTION
}

RÉSUMÉ : L'article interroge les entrecroisements et les rapprochements entre la poésie de Verlaine et la poésie chinoise, qui se révèlent dans la réception des poèmes de Verlaine en Chine des années 1910 aux années 1930. L'importation des poèmes de Verlaine se mêlait aux enjeux de l'évolution de la poésie chinoise, qui à cette époque, cherchait sa modernisation et était sensible à la question du rejet ou de l'héritage de la tradition. D'un côté, Verlaine était reçu comme un révolutionnaire pour la liberté de la versification par les Chinois qui voulaient se débarrasser de la prosodie canonique ancienne. De l'autre côté, aux yeux des hommes de lettres chinois les poèmes de Verlaine présentaient une qualité poétique - la suggestion, qui selon eux était une tradition dans la poésie chinoise ancienne, et qu'ils voulaient reconstituer dans la nouvelle poésie chinoise. Bian Zhilin a traduit un texte de Harold Nicolson à propos de l'intimité et la suggestion dans la poésie verlainienne, avec l'intention de remettre en valeur ces deux qualités poétiques qu'il trouvait inhérentes à la poésie chinoise ancienne. Cette idée a été soutenue par Qian Zhongshu. L'article tente de justifier ce rapprochement à l'appui des théories poétiques chinoises anciennes.

MOTS-CLÉS : Paul Verlaine ; poésie chinoise ancienne ; réception ; théorie poétique chinoise ; Bian Zhilin ; suggestion.

\section{THE POETRY OF VERLAINE AND THE CHINESE POETRY IN THE PERSPECTIVE OF THE RECEPTION}

\begin{abstract}
The article questions the intersections and the similarities between Verlaine's poetry and Chinese poetry, which were revealed in the reception of Verlaine's poems in China from the 1910s to the 1930s. The importation of Verlaine's poems was involved in the stakes of the evolution of Chinese poetry, which at that time was seeking its modernization and was sensitive to the question of rejection or the legacy
\end{abstract}

\footnotetext{
${ }^{1} \mathrm{Ye} \mathrm{Xu}$, doctorante à l'École normale supérieure de Paris, depuis 2017 sous la direction de M. Michel Espagne (ENS ULM) et la codirection de Mme Sophie Basch (Sorbonne Université).

Jangada | ano 9, nr. 17, jan/jun, 2021 | ISSN 2317-4722 


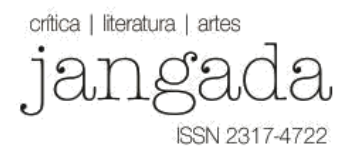

of tradition. On the one hand, Verlaine was received as a revolutionary for the freedom of versification by the Chinese who wanted to get rid of the old canonical prosody. On the other hand, in the eyes of Chinese men of letters, Verlaine's poems exhibited a poetic quality - the suggestion, which they said was a tradition in ancient Chinese poetry, and which they would reconstitute in new Chinese poetry. Bian Zhilin translated a text of Harold Nicolson about the intimacy and the suggestion in verlainian poetry, with the intention of re-emphasizing these two poetic qualities which he found inherent in the ancient Chinese poetry. This idea was supported by Qian Zhongshu. The article attempts to justify this similarity in support of ancient Chinese poetic theories.

KEYWORDS: Paul Verlaine; ancient Chinese poetry; reception; Chinese poetic theory; Bian Zhilin; suggestion.

\section{VERS UNE LITTÉRATURE MODERNISÉE}

La littérature chinoise des années 1910 aux années 1930, parmi de grands changements sociaux et bouleversements politiques, connut aussi de considérables évolutions, qui se manifestaient par une floraison des explorations littéraires engagées par les hommes de lettres de l'époque. Ce mouvement visant à construire une nouvelle littérature, composée néanmoins de nombreux courants différents, recouvrait différents aspects, allant des développements de nouveaux styles et formes littéraires, aux réflexions profondes et aux polémiques productives sur les théories littéraires, en passant par la prolifération des œuvres, écoles et journaux littéraires.

Cette évolution de la littérature faisait en fait partie des échos du Mouvement de la Nouvelle Culture $^{2}$ déclenché en 1915, un mouvement qui mit en question la culture traditionnelle chinoise et réclama une modernisation culturelle appuyée sur la science et la démocratie. La distance prise vis-à-vis de la tradition au niveau de la politique, de l'idéologie et des mœurs n'épargna pas la littérature : c'est dans ce contexte que les hommes de lettres se mirent à explorer de nouvelles voies littéraires adaptées à une Chine modernisée. Au centre des préoccupations liées à la construction de cette nouvelle littérature, se présentaient la question du rejet ou de l'héritage de l'ancienne littérature, et la question de l'assimilation de la littérature étrangère comme ressource nouvelle. Les lettres chinoises de cette époque furent profondément marquées par une multiplicité de traductions

\footnotetext{
2 新文化運動 (Xinwenhua Yundong)

Jangada | ano 9, nr. 17, jan/jun, 2021 | ISSN 2317-4722 


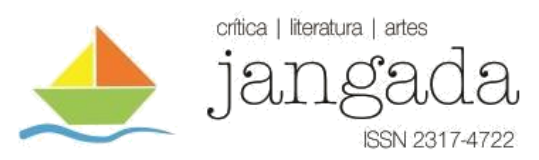

et articles de présentation pour les œuvres étrangères, et leurs influences s’intégrèrent dans la couche la plus fondamentale de la littérature chinoise contemporaine.

Sur l'aspect de la poésie, l'importation de la poésie symboliste française joua un rôle essentiel dans cette période de tâtonnement des poètes chinois, ce dont témoignaient la réception et l'influence de la poésie de Verlaine. Mais la littérature étrangère - dont les œuvres de Verlaine-, fut reçue et interprétée dans la perspective des partis pris spécifiques des littérateurs chinois de l'époque ; il s'agissait souvent des partis pris associés aux hésitations entre la tradition chinoise et la modernité chinoise. L'importation de la poétique étrangère n'était pas seulement une ouverture de la Chine à l'Occident, mais elle faisait aussi partie intégrale de l'évolution de la poésie chinoise elle-même. Le cas de la réception des œuvres de Verlaine était bien un exemple de cette rencontre entre Occident et Orient et de cette préoccupation entre tradition et modernité.

\section{LA LIBÉRATION DE LA VERSIFICATION ET L'ART DE LA SUGGESTION}

Des années 1910 aux années 1930 la traduction et la présentation de la poésie de Verlaine soulevèrent principalement deux questions - la libération de la versification et l'art de la suggestion. Dans les premiers articles d'introduction de Verlaine parus dans les années 1920, Verlaine était présenté comme le poète-réformateur qui avait lancé la libération de la versification dans la poésie française. L'article le plus important sur ce point fut «La prosodie de la poésie française et sa liberté $^{3} \gg$ de Li Huang ${ }^{4}$ paru en 1921. L'auteur y expliquait comment Verlaine se rebellait contre l'alternance des rimes féminines et masculines, contre l'exclusivité des vers pairs, contre l'interdiction du hiatus etc. Ce genre de discours revinrent sans cesse ${ }^{5}$, et l'image de Verlaine comme combattant de la liberté dans la versification était marquée.

\footnotetext{
${ }^{3}$ 《法蘭西詩之格律及其解放》 (Falanxi Zhi Gelü Jiqi Jiefang) La traduction de cette référence et celles des références et citations suivantes sont réalisées par l'ateur de l'article $\mathrm{Ye} \mathrm{Xu,} \mathrm{sauf} \mathrm{des} \mathrm{cas} \mathrm{suivis} \mathrm{d'une} \mathrm{note} \mathrm{supplémentaire.}$

4 李璜 (1895-1991)

${ }^{5}$ Voir l'article de Liu Yanling (劉延陵) 《Le symbolisme de la poésie française et les vers libres 》 (法國詩之象徵主義與自 由诗 Faguoshi Zhi Xiangzheng Zhuyi Yu Ziyoushi) (1922), l'ouvrage de Zhen Zhenduo (鄭振鐸) Les principes de la littérature (文學大綱Wenxue Dagang) (1927 : 1627), et l'article de Zhang Ruoming (張若苦) 《Les trois grands poètes du symbolisme français : Baudelaire, Verlaine et Rimbaud»(法國象征派三大詩人鮑德萊爾, 魏爾萊諾與懶苍 Faguo Xiangzhengpai San Da Shiren Baodelaier, Weierlainuo Yu Lanbao) (1937).

Jangada | ano 9, nr. 17, jan/jun, 2021 | ISSN 2317-4722

159 | P ág i n a
} 


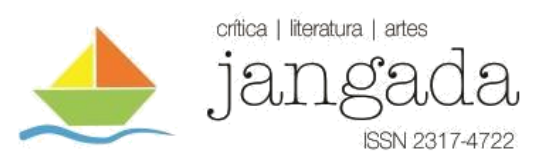

Le fait que Verlaine ait contribué à la liberté métrique correspondait bien au besoin des poètes chinois modernes de se débarrasser de l'ancien système de versification. Un des buts majeurs des mouvement littéraires de cette époque, était de remplacer dans l'écriture le chinois classique $^{6}$ par le chinois vernaculaire ${ }^{7}$, c'est-à-dire une écriture basée sur le mandarin parlé. La poésie moderne, née dans ce contexte, était censée renoncer à toutes les règles prosodiques canoniques et constitutives de la poésie ancienne, ainsi qu'à employer des vers libres et une syntaxe prosaïque ou même orale. Les jeunes poètes d'esprit réformateur se complaisaient à rappeler la tendance libératrice de la versification dans la poésie du symbolisme, et les vers libres promus par les symbolistes semblaient attribuer, selon eux, une légitimité à la révolution contre l'ancienne versification en Chine.

Au-delà de la liberté de la versification, les lecteurs chinois mirent aussi l'accent sur l'art de la suggestion dans les poèmes de Verlaine. Dans l'article «Deux grandes œuvres du poète Verlaine $^{8}$ » paru en 1922, l'auteur Wang Duqing 9 écrivit : « l'effet de la poésie symboliste consiste dans la suggestion ${ }^{10}$. » En 1930 Xiao Shijun ${ }^{11}$ traduisit et publia l'article d'Arthur Symons « Paul Verlaine », qui avait été publié en anglais en 1892. Cet article traduit attira le premier l'attention des Chinois sur la question de la suggestion chez Verlaine : «Tout est la suggestion, l'évocation — « des beaux yeux derrière des voiles » — la suggestion et l'évocation de sensations, la recherche sans cesse et insistante de la moindre nuance fine de l'expression ${ }^{12}$.» (1892) La suggestion verlainienne était souvent évoquée dans son rapport avec la «nuance ${ }^{13}$ », comme il s'agissait souvent d'un art de suggérer des choses qui, étant trop subtiles, échappent à l'expression discursive. Ainsi Symons rajoutait dans son article : «La nuance - quelque chose d'évanescent qui hante et taquine le cerveau de l'artiste, quelque chose qui ne peut pas, mais qui doit être exprimé ${ }^{14}$ ! » (1892)

${ }^{6}$ 文言 (Wenyan)

7 白話 (Baihua)

8 《詩人魏萊奈之二大名作》 (Shiren Weilainai Zhi Er Da Mingzuo)

${ }^{9}$ 王獨清 (1898-1940)

10 象徵詩底作用, 全在一個暗示。

11 蕭石君

${ }^{12}$ It is all suggestion, evocation — "des beaux yeux derrière des voiles" - the suggestion and evocation of sensations, a restless, insistent search for the last fine shade of expression.

${ }^{13}$ Shade

${ }^{14}$ La nuance - the something evanescent, which haunts and teases the artist's brain, the something which cannot, which must, be expressed.

Jangada| ano 9, nr. 17, jan/jun, 2021 | ISSN 2317-4722

160 | P ág i n a 


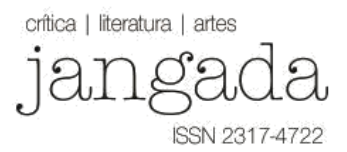

Dans l'article «Le symbolisme de la poésie française et les vers libres » écrit par Liu Yanling ${ }^{15}$, le critique mit aussi l'accent sur la nuance dans la poésie de Verlaine : «En somme, la nuance des sens et la représentation de l'impression sont deux choses qu'il a apportées au symbolisme ${ }^{16}$. » (1922)

La contribution qui se concentrait le plus sur cette question fut l'article «Verlaine et symbolisme $^{17}{ }$ du poète Bian Zhilin ${ }^{18}$ paru en 1932. Cet article était en fait la traduction d'un extrait tiré de l'ouvrage du diplomate et critique anglais Harold Nicolson, Paul Verlaine publié en 1921 à Londres — une biographie de la vie du poète suivie des critiques sur sa poésie. L'extrait traduit par Bian Zhilin visait à démontrer que l'intimité et la suggestion constituaient deux caractéristiques principales de la poésie verlainienne, et plus généralement de la poésie symboliste.

Il faut noter que la traduction de ce texte d'Harold Nicolson concernant l'intimité et la suggestion venait plus d'une réflexion que d'un choix aléatoire. D'abord, la poésie de Verlaine exerça des influences directes et profondes sur les poèmes et les pensées poétiques de Bian Zhilin. Celui-ci a écrit que depuis 1930 il avait commencé à lire les œuvres de Baudelaire, des Parnassiens, de Verlaine, de Mallarmé et des autres poètes français ${ }^{19}$ (1949), et que la traduction de l'extrait de Harold Nicolson et de certains poèmes de Baudelaire lui avaient apporté beaucoup d'inspirations sur son propre style poétique dans les années 1930 et $1940^{20}$ (2018, p. 339). Il est intéressant de comprendre la raison pour laquelle la poésie de Verlaine et ce texte de Nicolson attirèrent l'attention particulière de Bian Zhilin. La réponse à cette question semble être donnée dans l'épigraphe que le traducteur ajoutait au texte de Nicolson :

Pourquoi la poésie de Verlaine correspond particulièrement au goût des Chinois ? Est-ce que la poésie du symbolisme consiste dans l'accumulation des substantifs abstraits? Ces questions pourraient trouver un début de réponse

\footnotetext{
${ }^{15}$ Voir la note 5.

16 總而言之, 氣味底渾漠然, 情調底抒寫, 就是他捐助於象徵主義的兩件。

17 《魏爾倫與象徵主義》(Weierlun Yu Xiangzheng Zhuyi)

18 六之琳 (1910-2000)

19 恰巧因為讀了一年法文, 自己可以讀法文書了, 我就在 1930 年讀起了波德萊爾、高蹈派詩人、魏爾倫、瑪 拉美以及其他象征派詩人。

20 這些不僅多少影響了我在即 30 年代的詩風, 而且大致對三四十年代一部分較經得起時間考驗的新詩篇的產 生起過一定的作用。

Jangada | ano 9, nr. 17, jan/jun, 2021 | ISSN 2317-4722

161 | P á g i n a
} 


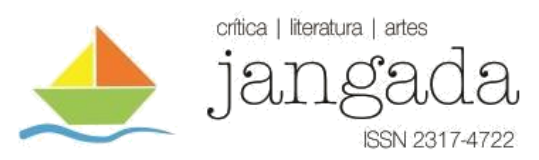

dans cet article, quoiqu'il ne soit pas écrit pour nous. [...] En réalité, le discours de Nicolson dans cet article n'est pas, dans le contexte chinois, nouveau. Intimité et suggestion, ne seraient-ce pas là précisément les qualités premières de la poésie ancienne chinoise ? Pourtant, il est probable que ces qualités soient bientôt oubliées par la plupart de nos poètes contemporains - ou peut-être le sont-elles déjà ${ }^{21}$. (2017, p. 248)

Curieusement, ces phrases de Bian Zhilin voulaient établir une affinité en termes d'intimité et de suggestion, entre la poésie de Verlaine et la poésie chinoise - précisément la poésie chinoise ancienne. En revanche, Bian Zhilin faisait allusion à la perte regrettable de ces deux qualités poétiques dans la poésie chinoise contemporaine. Dans ce discours, l'Occident et l'Orient se rencontraient, et à cette rencontre se mêlaient les enjeux de la poésie chinoise, laquelle voulait quitter la tradition et chercher une nouvelle modernité. Donc, cette épigraphe montrait que l'intention de la traduction du texte de Nicolson consistait à réclamer la récupération de ces deux qualités poétiques, qui selon Bian Zhilin se trouvaient chez Verlaine, et dans la Chine ancienne.

La mise en avant de la suggestion chez Verlaine dans les années 1920 et 1930 avait son contexte spécifique. Au début du mouvement de la Nouvelle Littérature, les précurseurs, par exemple $\mathrm{Hu} \mathrm{Shi}^{22}$, tendaient à protester intensément contre la poésie classique -- au niveau de la versification, de la syntaxe, de la rhétorique, de la sémantique, et de la thématique; et ils préconisaient pour la poésie des tonalités comme dans la prose ou même dans la parole, valorisaient l'expression directe des pensées et des sentiments, et appréciaient la description objective des choses. Cela délivra les poètes chinois du joug de l'ancienne formulation poétique et du chinois classique, mais en même temps cela poussa assez brutalement la poésie vers un prosaïsme ultérieurement considéré comme immature et vulgaire. Cette situation commença à changer au cours des années 1920 avec la naissance des poètes chinois revendiqués «symbolistes », qui à l'image du symbolisme français, cherchaient une «poésie pure », et favorisaient des modalités de

\footnotetext{
21 魏爾倫的詩歌為什麼特別合中國人的口味? 象征派做詩是不是只要堆砌一些抽象的名詞? 這種問題在這篇 文章里可以找出一點兒解答, 雖然這篇文章並非為我們寫的。[…其實尼柯孫這篇文章里的論調, 搬到中國來, 應當是並不新鮮, 親切與暗示, 還不是舊詩詞的長處嗎? 可是這種長處大概快要一或早已一被當代一般新詩人 忘掉了。

22 胡適 (1891-1962)

Jangada | ano 9, nr. 17, jan/jun, 2021 | ISSN 2317-4722

162 | P á g i n a
} 
lyrisme détournées de l'expression discursive, appuyées sur des analogies et métaphores, très implicites même un peu absconses. C'était pour éliminer la tendance au prosaïsme chez leurs prédécesseurs, que les poètes des années 1920 soulignèrent la valeur de la suggestion dans la poésie. Mu Mutian ${ }^{23}$, un poète symboliste chinois de cette époque, parla de l'importance de la suggestion pour la poésie dans son fameux article «De la poésie ${ }^{24} »$ :

Le monde de la poésie est un monde de la subconscience. La poésie doit avoir une grande suggestivité. [...] la poésie doit suggérer le mystère de la vie intérieure de l'humain. La poésie est suggestive et elle doit éviter l'explication. L'explication relève du monde de la prose ${ }^{25}$. (1926)

Ces efforts pour établir la suggestion dans la nouvelle poésie chinoise furent poursuivis par les poètes des année 30, en particulier ceux de l'école du Modernisme, à laquelle Bian Zhilin appartenait. Mais Bian Zhilin ne voyait pas la suggestion seulement dans le symbolisme importé : il pensait que celle-ci se trouvait aussi dans la poésie ancienne chinoise rejetée. Lorsque Bian Zhilin regrettait que l'intimité et la suggestion anciennes fussent «oubliées par la plupart de nos poètes contemporains - ou peut-être le sont-elles déjà », sans doute faisait-il référence au prosaïsme poétique imposé par ses aînés. Pour lui, le besoin de développer une poésie suggestive devrait aller de pair avec un retour à la tradition, ce que la poésie de Verlaine ou plus généralement du symbolisme mettait en lumière.

\section{PROBLÉMATIQUES CROISÉES : L'IMPORTATION OCCIDENTALE ET L'HÉRITAGE CHINOIS}

Des échos entre le symbolisme français et la poésie chinoise ancienne furent évoqués à plusieurs reprises par Bian Zhilin dans ses écrits ultérieurs, ce qui montrait que la traduction du texte de Harold Nicolson faisait en fait partie intégrale de ses considérations constantes et profondes sur ce

\footnotetext{
23 穆木天 (1900-1971)

24 《譚詩》 (Tan Shi)

25 詩的世界是潛在意識的世界。詩是要有大的暗示能 [...] 詩是要暗示出人的內生命的深秘。詩是要暗示的, 詩最忌說明的。說明是散文的世界的東西。

Jangada | ano 9, nr. 17, jan/jun, 2021 | ISSN 2317-4722 


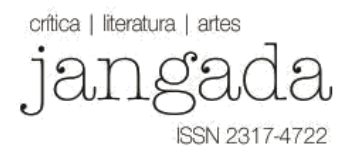

sujet. À propos de la présentation de la poésie symboliste française par le poète Li Jinfa ${ }^{26}$, Bian Zhilin écrivit :

Il a premièrement importé la poésie française du symbolisme de la deuxième moitié du XIX ${ }^{\mathrm{e}}$ siècle, et ses procédés et styles qui correspondent exactement à un des courants de la tradition dans la poésie ancienne chinoise ${ }^{27}$. (2018, p. 293)

Dans un autre article, Bian Zhilin exprimait le fait que l'assimilation du symbolisme français et le retour à la tradition chinoise avaient exercé leurs influences croisées sur ses propres poèmes, ce qui faisait entendre un écho entre ces deux systèmes poétiques :

Après que j'ai appris durant un an la langue française, mon intérêt dans la création poétique s'est orienté vers la piste de l'assimilation de la poésie traditionnelle chinoise. C'est au bon moment que j'ai pu prendre pour référence les poèmes du symbolisme qui étaient en grande partie français ${ }^{28}$. (2018, p. 338)

Jusqu'à la fin de sa vie, Bian Zhilin pensait que ses poèmes étaient à la fois occidentaux et traditionnels : « Si on dit que mes poèmes modernes sont occidentalisés, [...] ils n'en sont pas moins archaïsés. La première influence est formelle, visible, la seconde est spirituelle et insaisissable ${ }^{29} . »(2018$, p. 372)

Dans un article consacré à la commémoration de Liang Zongdai ${ }^{30}$ qui était poète, traducteur et critique de sa génération, Bian Zhilin indiquait qu'à peine connaissait-il la poésie française du symbolisme à travers des traductions et des œuvres d'imitation, qu'il y avait remarqué

\footnotetext{
26 李金髮 (1900-1976)

27 他為首引進19世紀後半期法國象征派詩風, 它和中國舊詩中的一路傳統恰好契合的手法以及同手法俱來的 風味。

28 學了一年法文以後, 寫詩興趣已轉到結合中國傳統詩的一個路數, 正好借擥以法國為主的象征派詩了。

29 我寫白話新體詩, 要說是“歐化” [...] 那麼也未嘗不“古化”。一則主要在外形上, 影響容易看得出, 一則完全 在內涵上, 影響不易著痕跡。

30 梁宗岱 (1903-1983)

Jangada | ano 9, nr. 17, jan/jun, 2021 | ISSN 2317-4722

164 | P á g i n a
} 


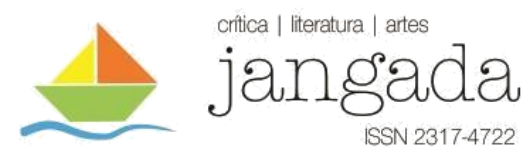

des échos stylistiques à la poésie chinoise traditionnelle ${ }^{31}(2018$ : 326). De plus, cet article montrait que la vision qui introduisait la poésie du symbolisme dans la question de l'héritage chinois, avait été en fait en même temps partagée par Liang Zongdai. Bian Zhilin résumait ainsi les idées de Liang sur cette question :

Une dizaine d'années après sa naissance, la nouvelle poésie avait une tendance différente ou même opposée par rapport à la tendance du premier moment. Pour en comprendre les raisons, il indiquait que les premiers essais prometteurs de la nouvelle poésie ne révoltaient pas seulement contre l'ancienne poésie, [...] ils étaient même ennemis de la poésie en général. Évidemment ce chemin n'était pas adéquat. Cependant, en raison des connaissances et des considérations plus profondes de la poésie occidentale, les successeurs ont compris davantage la valeur de l'héritage de la poésie ancienne pour la nouvelle. Ainsi l'écriture des nouveaux poèmes a eu des développements surprenants $^{32}[\ldots](2018$, p. 325)

Pour Bian Zhilin et Liang Zongdai, une des raisons d'apprendre les poèmes du symbolisme consistait à retrouver ce qui avait été dans la tradition poétique de la Chine mais qui avait été rejeté brutalement par les prédécesseurs de la nouvelle littérature. Bian et Liang relevaient effectivement d'une génération dont les pensées faisaient se chevaucher les influences occidentales et les traditions chinoises, et ainsi qui tendait à faire communiquer ou fusionner les deux ressources dans la construction de la nouvelle poésie. Ce genre de réflexions était à la mode dans les années 1920 et 1930. Par exemple Zhou Zuoren ${ }^{33}$, un des intellectuels les plus importants de cette époque en Chine écrivit en 1926 :

${ }^{31}[\ldots]$ 接觸到一點作為西方現代主義文學先驅的法國象征派詩, 只感到 $[\ldots]$ 與我國傳統詩(至少傳統詩中的一 路), 頗有相通處 $[\ldots]$

32 新詩發軔十幾年的趨勢, 恰與初期的分道揚鏕以至於背道而馳。究其原因, 他指出新詩的倡導者不僅反舊詩, “簡直是反詩的”, 此路必然不通, 由於對西方詩“深一層”的認識, 有所觀照, 進一步了解舊詩、舊詞對於新詩應 具的繼承價值, 一般新詩寫作有了他所謂驚人的发展 [...]

33 周作人 (1885-1967)

Jangada | ano 9, nr. 17, jan/jun, 2021 | ISSN 2317-4722

165 | P ág i n a 


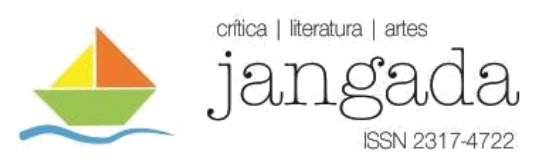

[...] Le symbole est le procédé le plus nouveau pour la poésie, mais c'est aussi le plus ancien, car il existe en Chine depuis l'antiquité. [...] C'est la nouvelle tendance à l'étranger, mais en même temps elle est aussi l'ancienne méthode en Chine. Si les nouveaux poèmes adoptent cette piste, la fusion aboutira et la vraie nouvelle poésie chinoise prendra naissance ${ }^{34}$. (1998, p. 174)

Donc, c'est sur le fond de ces questionnements qui mariaient l'importation occidentale et l'héritage chinois que Bian Zhilin traduisit le texte de Harold Nicolson à propos de l'intimité et la suggestion verlainiennes et lui accorda son épigraphe révélatrice. Ce texte et cette épigraphe nous aideraient à comprendre en quoi consistaient les affinités que les hommes de lettres chinois croyaient découvrir entre la poésie du symbolisme et la poésie chinoise ancienne.

\section{LA POÉSIE VERLAINIENNE LUE PAR NICOLSON : L'INTIMITÉ ET LA SUGGESTION}

Avant de s'interroger sur la légitimité de ce rapprochement jetant un pont de l'Occident à l'Orient ainsi que de l'antiquité à la modernité, il faudrait d'abord éclairer ce que Nicolson désigne par « intimité » et « suggestion ».

En fait le texte extrait et traduit par Bian Zhilin vient du chapitre VII du livre Paul Verlaine de Nicolson : ce chapitre est intitulé « La position littéraire de Verlaine ${ }^{35}$ », et vise à montrer « dans quelle mesure, donc, les caractéristiques spécifiques du symbolisme doivent être attribuées à Paul Verlaine $^{36} »(1921$, p. 238). Lorsqu'il parle des caractéristiques principales dans la poésie verlainienne, c'est aussi, à priori, pour parler de l'ensemble du symbolisme : « [...] les deux qualités, c'est-à-dire l'intimité et la suggestion. Il est en effet légitime de soutenir que l'ensemble du symbolisme est basé sur et dérive de ces deux sources d'inspiration ${ }^{37}$. » (1921, p. 236) Et selon lui, ces deux qualités poétiques constituent la contribution principale de Verlaine au symbolisme.

\footnotetext{
34 象征是詩最新的寫法, 但也是最舊，在中古也“古已有之”。[… 這是外國的新潮流, 同時也是中國的舊手法; 新詩如往這一路去, 融合便可成功, 真正的中國詩也就可以產生出來了。

${ }^{35}$ Verlaine's litterary position

${ }^{36}$ How far, therefor, are de specific characteristics of the Symboliste theory to be traced to Paul Verlaine.

${ }^{37}[\ldots]$ the two qualities, that is, of intimacy and suggestion. It is legitimate indeed to contend that the whole of Symbolism is based upon and derives from these two fountain-heads of inspiration.

Jangada | ano 9, nr. 17, jan/jun, 2021 | ISSN 2317-4722

166 | P ág i n a
} 


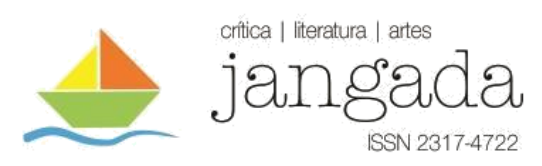

D'abord, l'intimité que Nicolson découvre chez Verlaine, est une sorte de qualité de la personne à la fois particulière et généralement humaine ${ }^{38}$ (1921 : 239) ; cet effet paradoxal entre l'individu et l'universalité est rendu par le mécanisme de l'association appuyé sur la référence aux objets insignifiants de la vie :

L'intimité des meilleurs poèmes de Verlaine n'est pas un exhibitionnisme inactif. Dans son essence il n'a rien comme les larmes de Musset, ni la vaine autobiographie de Hugo, ni l'esprit de catalogue de Balzac. Son effet réside d'abord dans l'utilisation parcimonieuse et habile des attributs, dans une référence apparemment fortuite mais vive à des objets mineurs qui rayonnent pour lui d'une signification émotionnelle. [...] notre sentiment d'association est mis en vibration par ces références, et une corde agréable est frappée par la pensée d'autres objets, intimes pour nous, qui ont précisément une telle connexion dans notre propre expérience ${ }^{39}$. (1921, p. 239)

D'ailleurs l'intimité verlainienne, Nicolson rajoute, se réalise aussi par des confidences bavardes $^{40}$ (1921 : 241) de sensations accidentelles dans les expériences triviales. Nicolson pose ce style en opposition au « grandiose » :

Il savait très bien que sa qualité poétique particulière n'était pas à l'écoute du grandiose, il savait que les émotions plus profondes lui échapperaient toujours.

Il préférait donc s'occuper des sensations plus accessoires qui reflètent les passions et les tragédies auxquelles sa vie se mêlait ${ }^{41}$. (1921, p. 242)

Il est intéressant de voir que dans ces deux éléments constitutifs de l'intimité verlainienne - l'association à travers les références aux objets mineurs et la confidence des sensations

\footnotetext{
${ }^{38} \mathrm{~A}$ feeling of a definite and immensely human personality

${ }^{39}$ The intimacy of Verlaine's better poems is no idle exhibitionism. It has nothing in its essence of the tears of de Musset, il has nothing of the vain autobiography of Hugo; it has nothing of Balzac's sense of catalogue. Its effect resides firstly in the sparing and skill use of attributes, in an apparently incidental but vivid reference to minor objects which for him radiate with emotional significance. [...] our sentiment of association is set vibrating by these references, that a pleasurable chord is struck by the thought of other objects, intimate to us, which have precisely such a connexion in our own experience.

${ }^{40}$ Garrulous confidences

${ }^{41} \mathrm{He}$ knew full well that his peculiar poetic quality was not attuned to the grandiose, he knew that the deeper emotions would always elude him, and he preferred, therefore, to deal with the more incidental sensation, reflect in them the passions and tragedies in which his life was involved.

Jangada| ano 9, nr. 17, jan/jun, 2021 | ISSN 2317-4722

167 | P á g i n a
} 


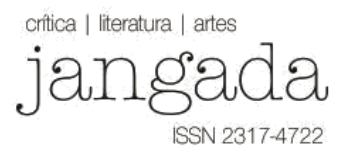

accidentelles - s'aperçoit déjà la suggestion, comme Nicolson l'indique : ce sont des détours vers l'intimitét2 (1921, p. 243). Il ne s'agit pas de l'épanchement direct, mais au contraire de la suggestion implicite à travers des choses concrètes de la vie.

À propos de la suggestion dans les poèmes du symbolisme, Nicolson précise dans la suite du texte :

Leur but, en effet, était de révéler l'infini, de construire une synthèse qui devait suggérer tout l'homme par l'art ; pour eux, ce qui est fatal dans la création, c'est le sens de la finalité : le chef-d'œuvre ne doit commencer que là où il paraît finir ; il ne doit pas simplement décrire, il doit suggérer ; il devrait laisser derrière lui des vibrations inexprimées. C'est ce sens de la suggestion, cette indication de l'intouchable, qui constitue la principale contribution de Verlaine à la nouvelle théorie ${ }^{43}$. (1921, p. 245)

Parmi tous les procédés de la suggestion de Verlaine, Nicolson met l'accent sur deux méthodes qui reviennent systématiquement dans ses œuvres. La première consiste à laisser au poème un horizon continu, infini, indécis dans les derniers vers : « dans de tels aperçus de l'infini, dans la simple habileté avec laquelle il frappe une corde vibrante dans les derniers vers de ses poèmes ${ }^{44} \gg(1921$, p. 247$)$.

La deuxième méthode que Verlaine emploie abondamment, rajoute Nicolson, est l'ouverture d'un poème par la représentation de la condition extérieure :

C'était une pratique constante pour lui d'ouvrir un poème avec une suggestion de conditions extérieures à travers lesquelles, par une vague transition, il expliquait et illustrait ses humeurs. [...] Cela lui permet de saisir la sensation fugace inspirée par un aspect soudain de la nature et de moduler son vers selon ces impressions ${ }^{45}$. (1921, p. 248)

\footnotetext{
${ }^{42}$ Indirect by-paths towards the intimate

${ }^{43}$ Their aim, indeed, was to reveal the infinite, to construct a synthesis which should suggest the whole of man by the whole of art; for them the fatal thing in creation was a sense of finality: the masterpiece should begin only where it appeared to end; it should not merely describe, it should suggest; it should leave behind it some unexpressed vibration. It is this sense of suggestion, this indication of the unattained, which constitutes Verlaine's chief contribution to the new theory.

${ }^{44}$ In such glimpses of the infinite, in the mere skill with which he strikes a vibrant chord in the las verses of his poems

${ }^{45}$ It was a constant practice of his to open a poem with a suggestion of exterior conditions through which, by some vague transition, he would explain and illustrate his moods. [...] It enables him to catch the fleeting sensation inspired by some sudden aspect of nature and to modulate his verse accordingly.

Jangada | ano 9, nr. 17, jan/jun, 2021 | ISSN 2317-4722

168 | P ág in a
} 
Il est essentiel de comprendre qu'ici ce qui suggère l'état d'âme n'est pas seulement une image d'ordre analogique, mais cette image analogique relève de l'environnement - souvent la nature, dans laquelle vit le poète ou la figure poétique, et qui suscite en celui-ci des sentiments et ainsi lui inspire la poésie : «Verlaine est en effet toujours sensible au « décor » dans lequel ses émotions ont été stimulées et a un don infaillible pour indiquer l'environnement naturel en quelques mots vifs ${ }^{46}$. » $(1921$, p. 249)

\section{L'INTIMITÉ DANS LA POÉSIE CHINOISE ANCIENNE}

À propos du rapprochement que Bian Zhilin éclaire, entre la poésie verlainienne - ou plus largement symboliste -- et la poésie chinoise ancienne sur les points communs de l'intimité et de la suggestion, il est intéressant de poursuivre la réflexion : est-il vrai que la poésie chinoise classique -- si ce n'est pas par son ensemble du moins par certains courants dans la tradition -- se caractérise par les même intimités et suggestion que Nicolson Harold trouve chez Verlaine ?

Avant tout la réponse est oui si l'on entend par «intimité » l'approfondissement dans l'exploration de l'intériorité humaine. En Chine, l'art du vers, depuis sa naissance et à travers toute son évolution, demeure principalement lyrique — il accorde toujours la première place aux sentiments intimes ${ }^{47}$. Mais comme ce que Nicolson démontre à propos de la poésie de Verlaine, les anciens poèmes chinois, eux-aussi, n'extériorisent pas les sentiments dans une exhibition directe : on y trouve très rarement les jérémiades lamartiniennes ou les exclamations hugoliennes, mais il y a une prédilection générale, comme chez Verlaine, à exprimer les sentiments en s'appuyant sur des références aux choses concrètes dans la vie banale.

\footnotetext{
${ }^{46}$ Verlaine is indeed always sensitive to the "décor" in which his emotions have been stimulated and has an unerring gift for indicating natural surroundings in a few vivid words.

${ }^{47}$ Voir La tradition du lyrisme de la littérature chinoise (中國文學的抒情傳統 Zhongguo Wenxue De Shuqing Chuantong) de Chen Shixiang 陳世襄 (2014).

Jangada | ano 9, nr. 17, jan/jun, 2021 | ISSN 2317-4722 


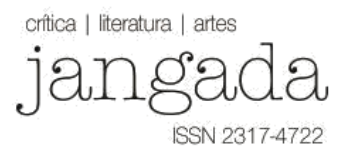

Les trois procédés rhétoriques, les plus anciens et les plus récurrents dans la littérature chinoise sont $f u, b i$ et $x_{i n g}{ }^{48}$. Selon $\mathrm{Zhu} \mathrm{Xi}^{49}$-- le plus important commentateur des classiques confucianistes, seul le $f u$ concerne l'expression $\operatorname{directe}^{50}$ (1991, p. 2), mais le bi, «c'est établir l'analogie entre une chose et une autre chose ${ }^{51} »(1991$, p. 3), et le xing, «c'est évoquer d'abord d'autres choses pour soulever les paroles à exprimer ${ }^{52} \gg(1991$, p. 1). Dans Le cour de la littérature et la sculpture des dragons ${ }^{53}$-- l'ouvrage de théorie littéraire le plus important en Chine vers 500, l'auteur Liu Xie ${ }^{54}$ consacre un chapitre entier à « bi et $x i n g »$ :

Le $b i$ procède par rapprochement et le xing par soulèvement. À propos du rapprochement d'idées, il s'agit d'utiliser l'analogie pour indiquer des faits ; à propos du soulèvement des sentiments, il s'agit de s'appuyer sur la moindre chose pour élaborer des paroles. Quand les sentiments sont sollicités, voici le xing est créé ; quand les idées sont rapprochées, voici le bi est né55. (1964, p. 601)

Le $b i$ et le xing sont tous des pistes détournées, qui passent par des références concrètes et amènent à la fin à l'intimité sentimentale. Dans la poésie chinoise ancienne, parmi les motifs les plus récurrents pour transmettre les sentiments, il y a le changement de saisons, l'écoulement de l'eau, le séjour aux lieux étrangers, les feuilles ou fleurs mortes, le vent et la pluie, le départ de l'être aimé, son absence, la vie retirée dans la nature, la retrait dans un pavillon, l'arrivée du

\footnotetext{
${ }^{48} \mathrm{Fu}$ (賦), bi(比), xing(興), ces trois termes se trouvèrent premièrement dans Les Rites de la dynastie Zhou (周禮
} Zhouli) et puis furent repris par le Classique des vers (詩經 Shijing) commenté par Mao Heng et Mao Chang. Fu, bi et xing étaient considérés et employés dans les propos de critique comme trois procédés rhétoriques - cette idée fut définitivement résumée par Kong yingda (孔穎達) : «Fu, bi et xing sont des procédés que la poésie utilise. 》 (賦比 興是詩之所用) voir dans La Bibliothèque accomplie des Quatre Disciplines des Lettres (四庫全書 Siku Quanshu) vol.69 (2013, p. 120).

49 朱喜 $(1130-1200)$

50 賦者,敷陳其事, 而直言之者也。

51 比者, 以彼物比此物也。

52 興者, 先言他物以引起所詠之辭也。

53 文心雕龍 (Wenxin Diaolong)

54 劉勋 $(465-522)$

55 故比者, 附也; 興者, 起也。附理者切類以指事, 起情者依微以擬議。起情故興體以立, 附理故比例以生。

Jangada| ano 9, nr. 17, jan/jun, 2021 | ISSN 2317-4722 


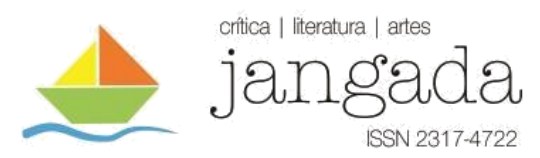

crépuscule etc.. Nous voyons que ces motifs préférés par les poètes chinoise relèvent tous de la vie quotidienne et presque insignifiante, mais c'est justement dans cette insignifiance que le sens émotionnel se manifeste. D'ailleurs c'est à travers les topoï de la banalité que tout le monde a connus, que l'association est établie entre le poète et les lecteurs, et qu'ainsi l'inimité pourrait être vraiment partagée.

Rappelons-nous que selon Nicolson, dans l'intimité verlainienne, ce qui va de pair avec les références aux choses insignifiantes est l'attachement aux sensations accidentelles : tout cela est selon lui en opposition au «grandiose ». Il s'agit d'un art consacré à la subtilité, à la frivolité, aux détails et aux nuances, ancré dans la vie ordinaire. Nous aurions évidemment tort de dire que toute la poésie chinoise classique est marquée par une extrême frivolité et subtilité des sentiments, parce que beaucoup de poèmes chinois renvoient aux sentiments grandioses, par exemple les poèmes qui regrettent l'évolution de l'histoire ou expriment des méditations existentielles.

Cependant, ce que Bian Zhilin voulait rapprocher de la poésie du symbolisme n'était pas l'ensemble de la poésie chinoise ; au contraire, il faisait attention à indiquer que c'est seulement « un des courants de la tradition dans la poésie ancienne chinoise ${ }^{56}$ », et préciser même la poésie « dans la tradition du $s h i^{57}$ de la dynastie Tang tardive et du $c i^{58}$ de la dynastie des Song du sud ${ }^{59}$ » (2018, p. 293). La poésie de ces périodes est justement marquée par la profusion de sensations accidentelles, souvent extrêmement subtiles, comme dans certains poèmes de Verlaine. Une fois Bian Zhilin a évoqué, au nom de la « décadence », cette ressemblance : « [...] dans mes poèmes de la première phase, il y avait même le même ton de décadence que dans la poésie de la dynastie Tang tardive et de la dynastie des Song du sud, qui ressemble un peu à l'ambiance de la poésie fin-

\footnotetext{
56 Voir la note 27.

57 詩. Shi, ce terme chinois fait référence aux œuvres littéraires qui sont caractérisées par les vers rythmés et par l'extériorisation des sentiments. Le shi est un genre littéraire chinois qui a de nombreuses formes différentes. Les formes les plus pratiquées dans l'histoire de la Chine est Jueju et Lüshi.

58 詞. Le $c i$ est une forme de la poésie chinoise, qui prospère pendant la dynastie Song. Le ci est écrit selon une mélodie codifiée, et chantée avec la musique. En raison de son attachement à la musique et de ses formes particulières, on a tendance à le distinguer des autres formes du shi.

59 晚唐詩, 南宋詞一路傳統. La dynastie Tang tardive va de 755 à 763 de notre ère, et la dynastie des Song du sud va de 1127 à 1279. Parmi les représentatifs du shi de la dynastie Tang tardive il y a Li Shangyin (李商隱), Du Mu(杜牧) et Wen Tingyun(溫庭筠). Parmi les représentatifs ou représentatives du $c i$ de la dynastie des Song du sud il y a Jiangkui(姜睡), Wu Wenying(吳文英), Li Qingzhao(李清照).
}

Jangada | ano 9, nr. 17, jan/jun, 2021 | ISSN 2317-4722

171 | P á g i n a 
de-siècle en Occident ${ }^{60}$. » $(2018$, p. 372) La subtilité sentimentale est justement une sorte de décadence, contrairement à ce qui est important, immense et impressionnant. Au demeurant, il faudrait comprendre « la poésie de la dynastie Tang tardif et de la dynastie des Song du sud » comme un style ou un courant poétique qui existe et évolue dans toute l'histoire de la poésie chinoise. Les époques de la dynastie Tang tardive et de la dynastie des Song du sud ne possèdent pas ce style exclusivement, mais elles sont juste considérées comme le moment de l'apogée dans le développement de ce style.

\section{LA SUGGESTION DANS LA POÉSIE CHINOISE ANCIENNE}

Dans la tendance à exprimer les sentiments de manière détournée à travers le $b i$ et le xing, nous voyons déjà la suggestion à l'œuvre dans la tradition poétique chinoise. La suggestion verlainienne, selon l'analyse de Nicolson, n'est pas seulement l'évocation d'un quelconque sentiment, mais c'est surtout la synthèse de sens qui rejoint l'infini. Qian Zhongshu ${ }^{61}$, un des plus grands critiques littéraires chinois au $\mathrm{XX}^{\mathrm{e}}$ siècle, dans son article « De la poésie chinoise ${ }^{62}$ », exprimait une opinion très proche de cette idée de Nicholson :

Les poètes chinois veulent, eux, montrer l'infini dans les limites. Un poète chinois a dit : «La parole est limitée, mais le sens est infini. » Un autre a dit : La meilleure poésie « peut décrire les paysages indicibles, qui apparaissent sous nos yeux ; elle peut exprimer des sens infinis, qui se révèlent en dehors de la parole. » Il s'agit de faire entrevoir dans une forme précise un état inexprimable, ce qui fait écho à l'art poétique de Verlaine : «Rien de plus cher que la chanson grise / Où l'Indécis au Précis se joint ${ }^{63}$. (1997a, p. 533)

\footnotetext{
${ }^{60}[\ldots]$ 我在前期詩的一個階段居然也出現過晚唐南宋詩詞的末世之音, 同時也有點近於西方“世紀末”詩歌的 情調。

61 錢鐘書 $(1910-1998)$

62 談中國詩 (Tan Zhongguo Shi)

63 簡短的詩可以有悠遠的意味, 收縮並不妨礙延长, 仿佛我們要看得遠些, 每把眉眼蘋歳。外國的短詩貴乎尖 刻斬截。中國詩人要使你從“易盡”里望见了“無垠”。一位中國詩人說:“言有盡而意無窮。”另一位詩人說 :

“狀難寫之景, 如在目前 : 含不盡之意, 见於言外。”用最精細確定的形式來逗出不可名言、難於湊泊的境界, 恰 符合魏爾蘭論詩的條件: 那灰色的歌曲, 空泛聯接著確切。
}

Jangada| ano 9, nr. 17, jan/jun, 2021 | ISSN 2317-4722 
Curieusement, Qian Zhongshu évoquait «Art poétique » de Verlaine pour expliquer la suggestion de sens infinis propre à la poésie chinoise. En fait, dans un autre article intitulé « La poésie chinoise et la peinture chinoise ${ }^{64} »$, Qian Zhongshu faisait référence aux opinions de trois critiques littéraire britanniques qui rapprochaient également la poésie chinoise de la poésie de Verlaine sur l'aspect de la suggestion :

Par exemple, quelqu'un a dit que la poésie chinoise ancienne est intangible, légère, suggestive, et que parmi les poèmes occidentaux, le plus proche de cela est Verlaine. Selon une autre personne, la poésie chinoise ancienne est simple mais révélatrice, et l'«Art poétique » de Verlaine pourrait servir de définition pour les principes de la littérature chinoise traditionnelle. Une autre personne a dit que les émotions lyriques dans la poésie chinoise ancienne ne sont jamais exprimées mais seulement suggérées ${ }^{65}$ [...] (1997b, p. 34)

Les méthodes de suggestion dans la poésie chinoise sont nombreuses: les sens se dissimulent derrière les métaphores, apparaissent en filigrane à travers des références culturelles, se projettent dans des paysages vagues, s'appuient sur des motifs stéréotypés, ou se laissent deviner dans l'ambiguïté langagière.

Nicolson écrit que les poèmes de Verlaine laissent souvent aux derniers vers une ouverture vers l'infini. En fait cette technique est appliquée partout dans les poèmes chinois : un vers subtil clôture le poème mais suggère pourtant un espace infini au-delà de la parole, comme le montrent les vers ci-dessous :

Le ciel m'avait en sa grâce accordé ta beauté,

Lune nocturne, voile de rêves vagues,

Vents printaniers, dix mètres de délicieux amour!

\footnotetext{
64 中國詩與中國畫 (Zhongguo Shi Yu Zhongguo Hua)

65 例如有人說, 中國古詩“空靈”, “清淡”, “含蓄”, 在西洋詩裡, 最接近韋爾蘭。另一個人, 中國古詩簡約隹永, 韋 爾蘭的《詩法》算得中國文學裡傳統原則的定義。還有人說, 中國古詩抒情, 從不明說, 全憑暗示 [...] Selon les notes dans l'ouvrage de Qian Zhongshu, les trois références sont les suivantes : «An Anthology » de Lytton Strachey, dans Characters and Commentaries ; «The Chinese Ideal » de Desmond Maccarthy, dans Experience ; «Windfalls » de R. C. Trevelyan.
}

Jangada | ano 9, nr. 17, jan/jun, 2021 | ISSN 2317-4722 
Mais le plaisir coule au fil de l'eau,

Le son des cordes est coupé, le parfum de soie est réduit.

Comment souffrir que des flocons de fleurs lutinent encore,

Et que des vapeurs de pluie câlinent le soleil couchant?

Languissant, les loriots font trainer des gazouillis ${ }^{66}$.

La méthode la plus commune que la poésie chinoise ancienne emploie pour la suggestion est aussi la plus importante chez Verlaine comme l'indique Nicolson : le commencement d'un poème par une suggestion de conditions extérieures dans lesquelles les sentiments sont stimulés.

Dans la poésie chinoise, il arrive fréquemment que les sentiments n'émergent pas seuls : ils résultent de l'incitation de l'environnement. Dans ce rapport entre l'environnement et le soi, le premier devient actif, le deuxième devient passif et les deux s'associent étroitement par un processus de stimulation-réaction : le soi se trouve exposé aux stimulations du monde extérieur, qu'il vit et sent; sous l'influence des éléments extérieurs, s'agitent en son cœur des vagues de sentiments.

Ce schéma est même inscrit dans les théories de la production littéraire depuis l'antiquité chinoise. Dans le chapitre «De la musique ${ }^{67} »$ du Classique des rites ${ }^{68}$, un des six classiques du confucianisme, il y a cette phrase : «Qu'un son se manifeste, il est né du cœur de l'homme. Les mouvements du cœur de l'homme, sont dus aux choses. Le cœur est agité par des choses et réagit, et ainsi il se manifeste par la voix ${ }^{69}$. $(1987$, p. 204) Cette phrase reconnaît manifestement que l'homme est l'objet d'une agitation des choses dans le monde extérieur, et cela amène des réactions chez lui, y compris l'émergence et l'expression des sentiments. Dans cette phrase, le mot essentiel

\footnotetext{
66 無端天與娉婷。夜月一簾幽夢, 春風十里柔情。怎奈向、歡娛漸隨流水, 素弦聲斷, 翠綃香減, 那堪片片飛花 弄晚, 蒙蒙殘雨籠晴。正銷凝。黃翮鳥又啼數聲。

67 樂記 (Yueji)

68 禮記 (Liji)

69 凡音之起, 由人心生也。人心之動, 物使之然也。感於物而動, 故形於聲。
}

Jangada | ano 9, nr. 17, jan/jun, 2021 | ISSN 2317-4722

174 | P ág i n a 
« $\operatorname{gan}^{70} »$, selon le dictionnaire Shuowen Jiezi $^{71}$ du II ${ }^{\mathrm{e}}$ siècle, signifie « agiter le cœur ${ }^{72} »(2017, \mathrm{p}$. 222). C'est à partir de ce sens fondamental que se développeront beaucoup d'autres sens de ce mot qui vont de percevoir sensiblement à avoir des émotions, jusqu'à être infecté.

Ce concept est ensuite renforcé et développé dans Le cour de la littérature et la sculpture des dragons $^{73}$ de Liu Xie. Dans le chapitre XLVI de l'ouvrage, intitulé « Couleurs de diverses $\operatorname{choses}^{74} »$, Liu Xie expose en détail comment les choses environnantes exercent leur influence sensiblement sur la mentalité de l'homme et ainsi lui inspirent la littérature :

Les quatre saisons se succèdent, le yin sévère et le yang clément s'altèrent ; auprès des mouvements des diverses choses du monde, le cœur bouge aussi. Quand l'air du yang émerge, les fourmis marchent, quand l'air du yin se condense, les oiseaux se nourrissent. Même les petits insectes sont agités, le mouvement des quatre saisons influence profondément les choses! Tandis que le jade précieux dresse son cœur intelligent, que la fleur jolie exhale son haleine pure, que les choses de diverses couleurs exercent des influences entre elles, qu'est-ce qui peut obtenir la tranquillité ? [...] chaque saison a ses choses particulières, chaque chose a ses manifestations particulières; les sentiments se transforment avec les choses, les mots s'expriment à partir des sentiments. Une seule feuille peut déjà amener des sentiments, les bruits d'insectes sont suffisants pour attirer le cœur, sans parler les nuits avec le vent et la lune, et les matins avec le soleil et le bois ${ }^{75}$ ! (1964, p. 693)

\footnotetext{
70 感

71 說文解字 (Explications étymologiques de la forme et de la prononciation des caractères)

72 感, 動人心也。

${ }^{73}$ Voir la note 53.

74 物色 (Wuse)

75 春秋代序, 陰陽慘舒, 物色之動, 心亦搖焉。蓋陽氣萌而玄駒步, 陰律凝而丹鳥盖, 微蟲猶或入感, 四時之動物

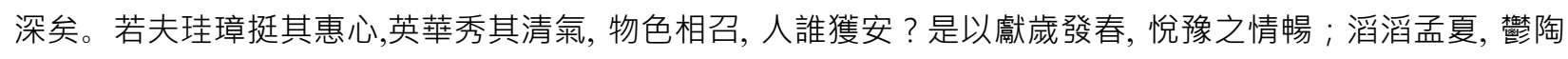
之心凝。天高氣清, 陰沉之志遠; 霞雪無垠, 矜肅之慮深。歲有其物, 物有其容; 情以物遷, 辭以情發。一葉且 或迎意, 蟲聲有足引心。況清風與明月同夜, 白日與春林共朝哉!
}

Jangada | ano 9, nr. 17, jan/jun, 2021 | ISSN 2317-4722 


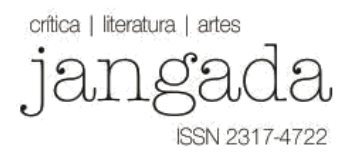

Zhong Rong ${ }^{76}$, un lettré important contemporain de Liu Xie, exprime la même idée dans sa fameuse Classification de la poésie ${ }^{77}$ : «Le souffle bouge les choses, les choses touchent l'homme, ainsi sa nature et son émotion s'agitent, ce qui se manifeste par la danse et la chanson ${ }^{78}$. » (2011, p. 1)

Si les théories littéraires présentées ci-dessus concernent plutôt le processus génétique par lequel la littérature doit se produire, en parallèle il y a aussi une figure rhétorique chinoise, qui consiste à mettre en place les provocations de l'environnement et les réactions de soi : c'est la figure de style xing, universellement et perpétuellement employé dans la poésie chinoise.

$\mathrm{Au}$ sens strict et dans la plupart des cas, le xing se situe aux premiers vers du poème et consiste à présenter l'image des choses - très souvent les paysages de la nature - qui stimulent des sentiments à exprimer dans la suite du poème, comme le montrent les trois exemples ci-dessous :

Au bord de l'eau crient les oiseaux ;

L'Homme a envie, de belles amies ${ }^{79}$.

Quelles Juaners ${ }^{80}$ exubérantes, j'en cueille et cueille

Mais elles ne remplissent jamais la corbeille.

Hélas! En la posant sur le grand chemin,

Je me rappelle mon amant lointain ${ }^{81}$.

La barque en cyprès flotte,

Au gré de l'eau qui court;

L'Homme stressé n'a pas de sommeil,

Avec du chagrin caché ${ }^{82}$.

\footnotetext{
76 鍾巆 (468-518)

77 詩品 (Shipin)

78 氣之動物, 物之感人,故搖蕩性情, 形諸舞詠。

79 關關睢鳩, 在河之洲; 窈察淑女, 君子好求。La traduction est réalisée par Yuanchong XU 許淵衝.

${ }^{80}$ Nom d'une plante.

81 采采卷耳, 不盈頃筐。嗟我懷人, 置彼周行。

82 泛彼柏舟, 亦泛其流。耿耿不槳, 如有隱憂。

Jangada| ano 9, nr. 17, jan/jun, 2021 | ISSN 2317-4722 
Cette figure de style, au niveau de la théorie ou de la pratique, correspond exactement à la suggestion de la condition extérieure au commencement du poème chez Verlaine. L'affinité est encore plus évidente si nous mettons en comparaison les vers chinois ci-dessus et le poème de Verlaine que Nicholson cite comme exemple sur ce point :

L'ombre des arbres dans la rivière embrumée

Meurt comme de la fumée,

Tandis qu'en l'air, parmi les ramures réelles,

Se plaignent les tourterelles.

Combien, ô voyageur, ce paysage blême

Te mira blême toi-même,

Et que tristes pleuraient dans les hautes feuillées

Tes espérances noyées !

D’ailleurs comme ce que Nicolson a trouvé chez Verlaine, le xing chinois introduit souvent un cadre environnant. Au-delà d'être image idéale dans le cadre du poème, les choses introduites par le xing en tête du poème sont souvent des objets ou paysages qui semblent avoir un contact sensible avec la figure du poème, et qui soulèvent des émotions. Le sens de « soulever » est même inhérent aux sens propres du mot xing, même en dehors de son usage comme figure de style. " Soulever $^{83}$ » est la signification que le dictionnaire Shuowen Jiezi ${ }^{84}$ donne au mot « xing » (2017, p. 54). Le philologue de la dynastie Tang Kong Yingda ${ }^{85}$ résume ainsi la fonction du xing dans son ouvrage Maoshi Zhengyi ${ }^{86}$ :

Le xing, c'est appuyer des faits sur des choses. Ainsi le xing, est le soulèvement.

Il porte sur l'analogie et la ressemblance, et aussi il s'agit d'inciter le cœur à

\footnotetext{
83 起

${ }^{84}$ Voir la note 71.

85 孔穎達 (574-648)

86 毛詩正義 (Commentaires pour les anciens commentaires du Classique des vers édité par Mao), est une œuvre de l'interprétation des anciennes notes canoniques du Classique des vers, voir dans La Bibliothèque accomplie des Quatre Disciplines des Lettres (四庫全書 Siku Quanshu) vol.69 (2013).

Jangada| ano 9, nr. 17, jan/jun, 2021 | ISSN 2317-4722 


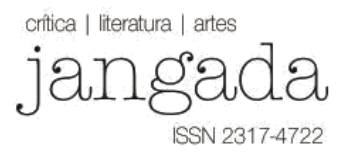

se manifester. Tous les vers dans le Classique des vers qui évoquent arbres, plantes, oiseaux et animaux, relèvent du $x$ ing $^{87}$. (2013, p. 120)

\section{LA POÉSIE DEVERLAINe REÇUe À TRAVERS LES PARTIS PRIS POÉTIQUES CHINOIS}

Le goût poétique chinois enclin à l'intimité et la suggestion qui est enraciné dans la tradition poétique, et l'envie de récupérer ces qualités dans la nouvelle poésie, contribuèrent beaucoup à donner une orientation pour la traduction des poèmes verlainiens en Chine des années 1920 aux années 1940. Les poèmes de Verlaine traduits et publiés pendant cette période, étaient presque tous les pièces emblématiquement marquées par l'intimité et la suggestion. Les traductions faites à partir de certains partis pris du goût poétique, et les articles de présentation qui mettent en avant certaines qualités de la poésie verlainienne, y compris le texte de Nicolson, présentèrent en fait une poésie de Verlaine que les lecteurs chinois de cette époque avaient envie de connaître et d'apprendre, mais qui ne correspondaient pas à la réalité de tout l'ensemble de la poésie verlainienne.

Comme Nicolson l'admet aussi dans son livre, les meilleures œuvres de Verlaine illustrant ces deux qualités sont dans ses premiers recueils. D'abord, les poèmes religieux à partir du recueil Sagesse s'éloignent visiblement de l'esprit de l'intimité mais s'approchent du grandiose en termes de thématique et de tonalité poétique; d'autre part vers la fin de sa vie, l'intimité devient l'épanchement confidentiel, de manière sincère, franche et directe, détaché au maximum des procédés artistiques : cette tendance est juste à l'antipode de l'art de la suggestion. Au fond, la poésie de Verlaine n'illustre pas l'intimité et la suggestion dans son ensemble, bien que ses premières œuvres offrent les meilleurs cristaux de ces deux éléments.

De plus, ce changement de style est une évolution volontaire de la part de Verlaine. Dans la préface à la première édition de Sagesse en 1881, Verlaine, sur le chemin vers le grandiose catholique, se moqua de la légèreté de ses anciennes œuvres : «L'auteur a publié très jeune, c'està-dire il y a une dizaine et une douzaine d'années, des vers sceptiques et tristement légers. Il ose compter qu'en ceux-ci nulle dissonance n'ira choquer la délicatesse d'une oreille catholique : ce serait sa plus chère gloire comme c'est son espoir le plus fier. » (2006, p. 66) Plus tard, Verlaine fit

\footnotetext{
87 興者, 託事於物, 則興者, 起也。取譬引類, 起發己心。詩文諸舉草木鳥獸以見意者, 皆興辭也。

Jangada | ano 9, nr. 17, jan/jun, 2021 | ISSN 2317-4722

178 | P ág in a
} 


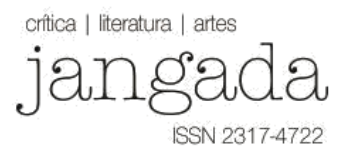

aussi une volte-face sur la question de la suggestion. Le 17 novembre 1884, il écrivit à Charles Morice : « la nécessité en certains cas de peindre net, fût-ce un peu avec son propre sang dilué dans ses propres larmes, quitte à être faux exprès et comédien en d'autres. » (2017, p. 22) L'envie de « peindre net », « annonce l'infléchissement de sa poétique vers l'objectivité, une des principales caractéristiques de sa production après Amour»(BIVORT, 2017, p. 23), et cela s'oppose évidemment à son esthétique de «nuance » théorisée par l'« Art Poétique ».

Les traductions des poèmes de Verlaine des années 1920 aux années 1940 offrirent aux lecteurs chinois une sélection venue principalement des premiers ouvrages du poète: Poèmes Saturniens, Fêtes Galantes, La bonne chanson et Romances sans Paroles. Par exemple, en 1921 Zhou $\mathrm{Wu}^{88}$ traduisit et publia «Chanson d'automne » et «Il pleure dans mon cœur »; en 1922 Tian han ${ }^{89}$ traduisit et publia « Chanson d'automne », « Il pleure dans mon cœur », « Après trois ans », « Nocturne Parisien », «Mon rêve familier », « Mandoline », « À Clymène », « Colloque Sentimental »; en 1922 Wang Duqing ${ }^{90}$ traduisit et publia «Chanson d'automne » et « La lune blanche »; en 1925 Li Sichun ${ }^{91}$ traduisit et publia « Chanson d'automne », « Le ciel est, par-dessus le toit », «Crépuscule du soir mystique », «Beams »; en 1926 Dai Wangshu ${ }^{92}$ traduisit et publia «Chanson d'automne » et «Il pleure dans mon cœur»; en 1930 Hou Peiyi ${ }^{93}$ traduisit et publia «Chanson d'automne », « La lune blanche», «Il pleure dans mon cœur », « Mon rêve familier » etc.. Les autres traductions pendant cette période favorisaient toujours les premières œuvres de Verlaine, et revenaient sans cesse aux pièces typiquement marquées par la suggestion et l'intimité, par exemple «Chanson d'automne » et «Il pleure dans mon cœur ».

Par rapport à la prédilection à ses premières œuvres de Verlaine, les œuvres des recueils après Sagesse, qui avaient, comme les œuvres des premiers recueils, contribué à la réputation de Verlaine en France, était pourtant complètement délaissées par les traducteurs chinois. C'est-à-dire, les traducteurs chinois ignorèrent consciemment ou inconsciemment les œuvres de Verlaine écrites

\footnotetext{
88 周無

89 田漢 (1898-1968)

90 王獨清 (1898-1940)

91 李思純 (1893-1960)

92 戴望舒 (1905-1950)

93 侯佩尹

Jangada | ano 9, nr. 17, jan/jun, 2021 | ISSN 2317-4722 


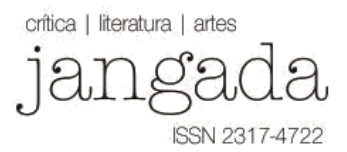

après son changement de style. Seul Li Jinfa ${ }^{94}$ introduisit dans son recueil de poèmes Petites Pluies $^{95}$ en 1925, ses traductions d'un poème d'Amours et d'un poème de Parallèlement, mais malheureusement ces deux pièces n'eurent pas de retentissement parmi les critiques et les traducteurs. Tout cela montre que la poésie de Verlaine fut reçue en Chine à travers de forts partis pris qui dérobaient aux lecteurs chinois le deuxième style de Verlaine : ce que les Chinois voyaient chez Verlaine, n'était que ce qu'ils souhaitaient connaître et apprendre - une poésie de l'intimité mêlée à l'art de la suggestion.

\section{RÉFÉRENCES BIBLIOGRAPHIQUES}

BIAN Zhilin (市之琳), «Verlaine et symbolisme» (魏爾倫與象徵主義). In : L'Intérieur et l'extérieur de la fenêtre vers l'occident (西窗內外). 廣州: 花城出版社, 1927.

BIAN Zhilin (市之琳), « Certaines réflexions sur mes expériences avant le séminaire sur la poésie anglaise »(開講英國詩想到的一些體驗), 文藝報. 北京, vol. 1-4, 1949.

BIAN Zhilin (六之琳), «Patriotisme et auto-théâtralisation : commémorer M. Ye Gongchao » (赤 子心與自我戲劇化 : 追念葉公超). In : Recueil d'articles de Bian Zhilin (市之琳集). 北京 : 中 國社會科學出版社, 2018.

BIAN Zhilin (市之琳), «Les fautes et les contributions de la traduction pour la poésie chinoise contemporaine »(翻譯對於中國現代詩的功過). In : Recueil d'articles de Bian Zhilin (市之琳 集). 北京: 中國社會科學出版社, 2018.

BIAN Zhilin (六之琳), « Préface pour les expériences d'un petit insecte » (雕蟲紀歷自序). In : Recueil d'articles de Bian Zhilin (市之琳集). 北京 : 中國社會科學出版社, 2018.

BIAN Zhilin (市之琳), «Les fatalités de la vie : commémorer M. Liang Zongdai »(人事固多乘 : 紀念梁宗岱). In : Recueil d'articles de Bian Zhilin (六之琳集). 北京 : 中國社會科學出版社, 2018.

BIVORT Olivier, «Introduction ». In : Paul Verlaine, Amour suivi de Parallèlement. Paris : Le livre de poche, 2017.

\footnotetext{
${ }^{94}$ Voir la note 26.

95 微雨

Jangada | ano 9, nr. 17, jan/jun, 2021 | ISSN 2317-4722
} 
CHEN Shixiang (陳世襄), La tradition du lyrisme de la littérature chinoise (中國文學的抒情傳 統). 北京 : 三聯書店, 2014.

DAI Wangshu (戴望舒), «Chanson d'automne» 《Il pleure dans mon cœur », 瓔珞. 上海, vol. 1, 1926.

HOU Peiyi (侯佩尹), « Chanson d'automne », « La lune blanche », « Il pleure dans mon cœur », «Mon rêve familier», 现代文学. 上海, vol. 1-5, 1930.

KONG Yingda (孔穎達), «Maoshi Zhengyi »(毛詩正義). In : Siku Quanshu (四庫全書) vol. 69. 台北: 商務印書館, 2013.

LI huang (李璜), 《La prosodie de la poésie française et sa liberté » (法蘭西詩之格律及其解放), 少年中國. 北京, vol. 2-21, 1921.

LI Jinfa (李金髮), Petites Pluies (微雨). 北京 : 北新書局, 1925.

LI Sichun (李思純), «Recueil de poèmes de la seine» (仙河集), 學衡. 北京, vol. 47, 1925.

LIU Yanling (劉延陵), «Le symbolisme de la poésie française et les vers libres »(法國詩之象徵 主義與自由诗), 詩.北京, vol. 1-4, 1922.

LIU Xie (劉勰), Le Cour de la littérature et la sculpturedes dragons (文心雕龍).香港 : 商务印 書館, 1964.

MU Mutian (穆木天), « De la poésie 》(譚詩), 創造月刊. 上海, vol. 1-1, 1926.

NICOLSON Harold, Paul Verlaine. London : Constable \& Co., 1921.

QIAN Zhongshu (錢鐘書), «De la poésie chinoise »(談中國詩). In : Essaies de Qian Zhongshu (錢鐘書散文). 杭州: 浙江文藝出版社, 1997.

QIAN Zhongshu (錢鐘書), « la poésie chinoise et la peinture chinoise » (中國詩與中國畫). In : Essaies de Qian Zhongshu et Yang Jiang (錢鐘書楊絳散文). 北京 : 中國廣播電視出版社, 1997. SYMONS Arthur, « Paul Verlaine », The National Review. London, vol. 19-112, 1892.

TIAN Han (田漢), «Pauvre Lélian 》 (可憐的侶離雀), 創造. 上海, vol. 1-2, 1922.

VERLAINE Paul, Sagesse. Paris : Le livre de poche, 2006. . Amour suivi de Parallèlement. Paris : Le livre de poche, 2017. 


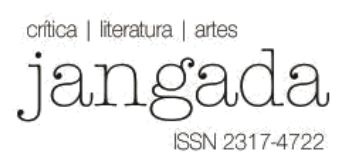

WANG Duqing (王獨清), 《Deux grandes œuvres du poète Verlaine 》(詩人魏萊奈之二大名 作), 學藝雜誌.上海, vol. 4-2, 1922.

XIAO Shijun (萧石君), «Verlaine»(魏尔伦), 文藝月刊. 南京, vol. 1, 1930.

XU Shen (許慎), Shuowen Jiezi (說文解字). 北京 : 中華書局, 2017.

ZHANG Ruoming (張若苦), «Les trois grands poètes du symbolisme français: Baudelaire, Verlaine et Rimbaud»(法國象征派三大詩人鮑德萊爾, 魏爾萊諾與懶苍), 中法大學月刊. 北 京, vol. 11-4.5, 1937.

ZHEN Zhenduo (鄭振鐸), Les principes de la littérature (文學大綱). 北京 : 商务印書館, 1927.

ZHONG Rong (鍾嵓), Classification de la poésie (詩品). 上海 : 上海古籍出版社, 2011.

ZHOU Wu (周無), «Chanson d'automne», «Il pleure dans mon cœur», 少年中國. 北京, vol. 2-9, 1921.

ZHOU Zuoren (周作人), «Préface à Yangbian $J i »($ (揚鞭集序). In : Anthologie des préfaces et postfaces pour la nouvelle poésie chinoise (中國新詩集序跋選). 廣州: 珠海出版社, 1998.

ZHU Xi (朱喜), Shijing Jizhuan (詩經集傳). 上海 : 上海古籍出版社, 1991.

Classique des rites (禮記). 上海: 上海古籍出版社, 1987. 